\title{
A LINGUAGEM DOCUMENTÁRIA NA NEGOCIAÇÃO DE UMA POLÍTICA DE INDEXAÇÃO PARA BIBLIOTECAS UNIVERSITÁRIAS: PROCEDIMENTOS E ESTRATÉGIAS DA PESQUISA-AÇÃO INTEGRAL'
}

The documentary language in the negotiation of an indexing policy for university libraries: procedures and strategies of integral research-action

\author{
Mariângela Spotti Lopes Fujita \\ Doutora em Ciências da Comunicação (USP). Professora \\ Titular (UNESP). Docente do Programa de Pós-Gradua- \\ ção em Ciência da Informação (UNESP/Marília). Bolsista \\ de Produtividade em Pesquisa 1C - CA AC.
}

RESUMO: A qualidade dos serviços de disseminação online da biblioteca depende da linguagem utilizada para a indexação e recuperação. A linguagem documentária é o instrumento de controle de vocabulário e de política de indexação que faz a diferença na mediação com a linguagem do usuário durante as estratégias de buscas em catálogos online que requerem especificidade e exaustividade. Considerando-se a problemática de uso e não uso de linguagem em catálogo de bibliotecas universitárias, a proposição desta investigação é a de demonstrar o processo de negociação, acerca da linguagem como instrumento facilitador da política de indexação realizado por sistema de biblioteca universitária com método de pesquisa-ação integral. O processo de negociação da política de indexação realizado com a participação de pesquisadores e catalogadores é relatado com enfoque nas decisões sobre a linguagem que produziram mudanças. A elaboração do manual de indexação inclui ações referentes à elementos, variáveis, processo de indexação e, principalmente, acerca do uso de uma única linguagem construída e atualizada pelos catalogadores. Conclui-se que o método de pesquisa-ação integral revelou as diferentes perspectivas dos participantes do contexto de indexação, usuários, catalogadores, dirigentes e bibliotecários sobre os problemas e restrições do contexto de bibliotecas, como seria possível resolvê-los e quais os instrumentos e métodos necessários, o que ajudou no aprimoramento da política de indexação.

PALAVRAS-CHAVE: Linguagem documentária. Política de indexação. Pesquisa-ação integral. 


\begin{abstract}
The quality of the online library services depends on the documentary language used for the indexing and information retrieval. The indexing language is the control tool of vocabulary and of indexing policy that makes the difference in mediation with the user language during the search strategies in online catalogues that require specificity and indexing depth. Taking into account the problem of use and no use of documentary language in catalogues of university libraries, the proposition of this research is to demonstrate the process of negotiation about the documentary language as facilitator of the indexing policy carried out by university library system with method of integral action-research. The process of negotiating the indexing policy carried out by researchers and cataloguers is reported with focus on the decisions about the documentary language that has produced changes. The development of the indexing manual includes actions related to elements, variables, indexing process and especially about the use of a single language constructed and updated by cataloguers. It is concluded that the method of integral researchaction revealed the different perspectives of the indexing process participants, users, cataloguers, managers and librarians about the problems and restrictions of libraries' context, how it would be possible to solve them and which were the necessary tools and methods, which helped in improving the indexing policy.
\end{abstract}

KEYWORDS: Documentary language. Indexing policy. Integral research-action.

\title{
1 Introdução
}

Quando se trata de biblioteca logo vem às nossas mentes seu catálogo e o acesso aos diversos documentos, pois é o instrumento Pesquisa desenvolvida com apoio da FAPESP e CNPq mais conhecido de recuperação da informação, o mais popular e o que mais divulga a representação de biblioteca no imaginário coletivo. Isso não é pouca coisa se considerar o global e o local sobre referentes universais e o catálogo da biblioteca, certamente, é um dos mais conhecidos.

É possível afirmar, contudo, que a forma de acesso ao catálogo de biblioteca mudou bastante em função do crescimento de quantitativo de itens bibliográficos, ou seja, era restrito ao local físico e atualmente é aberto de forma eletrônica e virtual pela internet; as buscas eram, em sua maioria, realizadas por autor e título são atualmente também por assuntos; o comportamento de busca do usuário exige mais precisão, exaustividade e especificidade à semelhança dos navegadores da internet.

A qualidade da indexação conduz à recuperação de informações úteis pelos usuários locais e remotos de bibliotecas. Essa afirmação tem respaldo no estudo teórico de Lancaster (2004) que expõe sobre a política de indexação ser determi- 
nante dos resultados quantitativos e qualitativos de consistência do processo de indexação e da precisão e revocação na recuperação da informação.

No contexto interdisciplinar de bibliotecas universitárias, investigações anteriores (FUJITA, RUBI, BOCCATO, 2009; FUJITA, BOCCATO, RUBI, 2012) indicam mudanças significativas no comportamento informacional de acesso e uso do catálogo por usuários que solicitam recuperação por assuntos com mais especificidade, compatibilidade com sua linguagem de busca e disponibilidade de mecanismos de interação tanto para o catalogador que indexa, quanto para o usuário que recupera.

Nesse contexto, a linguagem documentária é o instrumento que faz a diferença na mediação com a linguagem do usuário durante as estratégias de buscas em catálogos online. A qualidade dos serviços de disseminação online da biblioteca depende da linguagem utilizada para a indexação e recuperação porque não é possível a mediação física do bibliotecário, sobretudo quando a busca é por assuntos e exige mais precisão ou revocação com alta especificidade ou exaustividade.

Para que essa mediação aconteça é necessário que o bibliotecário realize o processo de indexação na catalogação com uso de uma linguagem documentária que seja a mesma a ser utilizada pelo usuário no momento da busca de modo a possibilitar o uso de uma mesma linguagem na representação da informação do conteúdo documental e na recuperação da informação. No entanto, a mediação realizada durante a busca por assunto em catálogos online implica a disponibilização efetiva da linguagem junto à interface de busca nos catálogos online, providência técnica esta que modifica sobremaneira o comportamento informacional dos usuários.

Essas condutas são determinantes para atingir o principal objetivo da representação da informação dentro do processo de indexação que é a recuperação da informação, porém, estão atreladas a uma política de indexação negociada com os componentes que realizam funções essenciais e interligadas para que a representação aconteça, quais sejam: o indexador, a linguagem documentária, elementos e variáveis da política de indexação da biblioteca ou serviço de informação. Isso significa que não basta ter uma linguagem se ela não for utilizada no processo de indexação na catalogação e, portanto, a recuperação não levará o usuário a acessar nenhum conteúdo significativo se o documento não foi representado com a mesma linguagem de busca.

A política de indexação pode ser definida por diversos fatores em uma unidade de 
informação, desde a seleção de tipos de documentos a serem indexados, procedimentos de análise e representação de assuntos, aspectos qualitativos da indexação como precisão, especificidade, exaustividade e revocação, instrumentos de controle de vocabulário tais como linguagem documentária ou opção por trabalhar com linguagem natural, uso de programas de indexação automática, além da avaliação da indexação pela consistência e pela recuperação.

Todos esses fatores, entretanto, ganham significado quando aplicados ao contexto da biblioteca universitária que possui finalidades e objetivos e abriga condições em seu ambiente quanto à natureza da informação produzida e solicitada, bem como características interdisciplinares da comunidade científica de seus usuários.

Considerando-se a problemática de uso e não uso de linguagem(ns) em catálogo de bibliotecas universitárias, a proposição desta investigação é a de demonstrar o processo de negociação, acerca da linguagem como instrumento facilitador da política de indexação, realizada por sistema de biblioteca universitária com método de pesquisa-ação integral. Tal negociação teve objetivo de alcance de exaustividade e especificidade na indexação e recuperação da informação em catálogo on-line.

\section{Linguagem documentária em bibliotecas: tipos e funções}

Para avançarmos no que tange ao uso da linguagem documentária em bibliotecas examinaremos este instrumento a partir de sua concepção como Lista de Cabeçalhos de Assuntos porque foi nesse ambiente que surgiram as listas de cabeçalhos de assuntos. A biblioteca, entendida como sistema de organização e recuperação da informação, possui contexto próprio em decorrência de sua organização e funções que são diversas de outros tipos de sistemas.

A origem remota das listas de cabeçalhos de assunto pode ser identificada nos índices alfabéticos de assunto que suplementaram, em muitos casos, os primitivos catálogos de autores. A seleção dos termos desses índices não obedecia a nenhum esquema. Durante muito tempo, para indicar o assunto de um livro, era considerado suficiente o emprego das próprias palavras do título, da mesma forma com que apareciam na folha de rosto, ou na forma invertida para destacar a palavra chave. Somente quando se verificou, na prática, a insuficiência desse método é que se procurou normalizar os critérios para utilização dos verdadeiros cabeçalhos. Surgiram, 
então, as listas, como uma decorrência lógica da experimentação prática, servindo como exemplo e sugestão ao trabalho da catalogação de assunto.

A escolha dos cabeçalhos de assunto, antes dos atuais catálogos on-line, era condicionada pela classificação, servindo mesmo de complemento à classificação dos livros nas estantes. O que a classificação separava, em seus diferentes aspectos, o cabeçalho tornava a unificar num só conjunto. Esta era a diferença fundamental entre os princípios da classificação e os da escolha dos cabeçalhos. Como característica dos cabeçalhos de assunto, destaca-se o seu aspecto de linearidade, o que o torna aplicável apenas a pesquisas unidimensionais.

O que caracterizava de modo mais marcante essas linguagens documentárias são os cabeçalhos de assunto. Os cabeçalhos de assunto apresentam uma gramática bem estruturada, com rígido controle de sinônimos, quase sinônimos, homógrafos, mas, uma grande limitação é que faziam referência (ver também) apenas do geral para o específico e de alguns assuntos correlatos, por isso, os cabeçalhos de assunto eram sistemas em que o princípio de hierarquia não era constante. Eram linguagens não-hierárquicas basicamente enumerativas.

Porém, com a evolução dos antigos catálogos de bibliotecas em catálogos online, as principais listas de cabeçalhos de assunto atualmente existentes introduziram estruturas hierárquicas com base em sistemas classificatórios, dos quais se originam, que produzem as relações hierárquicas entre os termos. Com isso, os termos de relações hierárquicas se diferenciaram dos termos de relações semânticas e associativas.

A Library Congress of Subject Headings - LCSH evoluiu em suas últimas edições com a introdução e uso da nomenclatura de relações semânticas adotadas por tesauros, incluindo uma estrutura lógica de relações hierárquicas anteriormente atrofiadas na linguagem. Considerando-se a influência que tal linguagem possui, sobretudo em catálogos de bibliotecas, é sintomático de que as mudanças foram operadas para que a recuperação por assuntos se tornasse mais precisa e específica. Tais mudanças influenciaram outras linguagens com formato de lista de cabeçalhos de assunto que adotaram características de tesauro.

As principais e mais gerais listas de cabeçalhos de assunto, atualmente existentes, são: a Library Congress of Subject Headings - LCSH, Sears List of Subject Headings, Lista de cabeçalhos de Assuntos da rede BIBLIODATA- LCARB, Terminologia de assuntos da Biblioteca Nacional do Brasil. 
Todas as linguagens citadas são atualizadas e utilizadas por várias bibliotecas no Brasil e no mundo cujos catálogos são acessíveis pela web. Além disso, é expressivo o número de descritores que possuem e os registros de autoridade correspondentes que servem à finalidade de compatibilização com uso do recurso eletrônico de interoperabilidade, ou seja, é possível outras bibliotecas construírem suas próprias linguagens realizando a importação desses registros de autoridade disponíveis em catálogos on-line. As duas últimas linguagens foram construídas no Brasil e são resultados de adaptação e tradução da LCSH.

Por isso existem linguagens que se intitulam listas de cabeçalhos de assunto e são utilizadas em sistemas pós-coordenados² como é o caso da Medical Subject Headings $(\mathrm{MeSH})^{3}$ onde sua condição de lista de cabeçalhos de assunto é negada, sendo tratado como tesauro, ou seja, oferecem possibilidades de síntese que nas listas mais tradicionais é representada pelos sub-cabeçalhos. Possuem paralelamente ao arranjo alfabético (usam termos da própria linguagem natural e por isso determinam uma ordenação alfabética), um arPós-coordenação: coordenação de termos na busca - Passa a idéia de uma linguagem onde o instrumento de controle de vocabulário exerce uma função sugestiva. ranjo sistemático ou facetado. Assim como o vocabulário estruturado Descritores em 3 Usado com o sistema MEDLARS

Ciências da Saúde (DeCS), utilizado para a indexação e recuperação da informação em fontes de informação disponíveis na Biblioteca Virtual em Saúde (BVS), foi desenvolvido a partir do MeSH.

Com esta mesma condição, outras linguagens surgiram com base no vocabulário de listas de assuntos compiladas pelas bibliotecas universitárias mais especializadas na elaboração dos catálogos de assuntos que tem uma mistura de princípios de cabeçalhos de assuntos e de tesauros tal como o Vocabulário Controlado do SIBI/ USP - VocaUSP que "é uma linguagem documentária construída, a partir de procedimentos terminológicos e documentários, pelos bibliotecários do SIBi/USP, com a participação de especialistas de todas as áreas do conhecimento abrangidas pelos seus descritores." (LIMA, BOCCATO, 2009).

É importante destacar o protagonismo da Terminologia de Assuntos da Biblioteca Nacional no cenário brasileiro de bibliotecas. Trata-se de um exemplo de linguagem a ser considerado tendo em vista a contínua atualização e adaptação da LSCH à terminologia usada em bibliotecas brasileiras, o que favorece as políticas de indexação para recuperação em catálogos online. 
Por outro lado, não é possível ignorarmos a significativa contribuição da Lista de Cabeçalhos de Assuntos da rede BIBLIODATA, atualmente sob responsabilidade do Instituto Brasileiro de Informação em Ciência e Tecnologia (IBICT), que a despeito do importante vocabulário construído ao longo de várias décadas, é uma linguagem documentária utilizada por várias bibliotecas brasileiras, universitárias, públicas, escolares e especializadas brasileiras, que não atualizou sua estrutura de relações entre termos tal como a LCSH e a Terminologia de Assuntos da Biblioteca Nacional.

Essa e outras questões que envolvem as linguagens documentárias é uma providência necessária que merece estudo no âmbito de uma política nacional de indexação e representação da informação.

\section{A pesquisa-ação integral na negociação da política de indexação para bibliotecas universitárias}

O processo de negociação da política de indexação é realizado, desde 2010, com catalogadores das bibliotecas universitárias Conta com o apoio da Coordenadoria Geral de Bibliotecas da UNESP à pesquisa “Política de indexação para bibliotecas" (Processo CNPq: 305648/2009-8 - BOLSA PQ nível 1C). da $\operatorname{UNESP}^{4}$ utilizando a metodologia da pesquisa-ação integral que os levou a decidir sobre a elaboração, manutenção e uso da Linguagem UNESP. Tal decisão tornou possível a oportunidade única de envolvimento cooperativo entre pesquisadora e participantes em torno da situação de elaboração, implantação, manutenção e uso da linguagem documentária.

O objetivo de formar equipe de catalogadores de bibliotecas universitárias para elaborar, usar e avaliar linguagem documentária foi realizado mediante aplicação da metodologia de pesquisa-ação para intervenção na realidade das bibliotecas como forma de melhor observar problemas e mudanças.

A pesquisa-ação é, segundo Thiollent (1998, p.14)

[...] um tipo de pesquisa social com base empírica que é concebida e realizada em estreita associação com uma ação ou com a resolução de um problema coletivo e no qual os pesquisadores e os participantes representativos da situação ou do problema estão envolvidos de modo cooperativo ou participativo.

A pesquisa-ação possui um ciclo composto de cinco etapas na visão de 
Descombe (2005 apud LIMA, 2007): a partir da prática profissional (1) é feita uma reflexão crítica (2) identificando o problema ou avaliando mudanças. Em seguida, ocorre a etapa da Pesquisa (3) realizada de forma sistemática e suas descobertas incorporadas em um plano de ação na etapa de planejamento estratégico (4) e, assim, as mudanças são implementadas na Ação (5), fase final do ciclo.

Morin (2004, p.56) compreende que o termo pesquisa-ação "designa em geral um método utilizado com vistas a uma ação estratégica e requerendo a participação dos atores". Nessa perspectiva participativa do método, propõe modalidades de pesquisa-ação com base no nível de participação dos atores definido por Desroche (1990, p.108) demonstrado no Quadro 1 abaixo:

\begin{tabular}{|c|c|c|c|c|}
\hline \multicolumn{5}{|c|}{ PESQUISA } \\
\hline & De explicação & De aplicação & De implicação & Tipo de partiicipação \\
\hline & $\begin{array}{c}\text { Sobre a ação e } \\
\text { seus atores }\end{array}$ & $\begin{array}{c}\text { Para a ação de } \\
\text { seus atores }\end{array}$ & $\begin{array}{c}\text { Pela ação e seus } \\
\text { atores }\end{array}$ & \\
\hline 1 & + & + & + & Integral \\
\hline 2 & + & + & - & Aplicada \\
\hline 3 & + & - & + & Distanciada \\
\hline 4 & + & - & + & Informativa \\
\hline 5 & - & + & + & Espontânea \\
\hline 6 & - & + & + & Usuária \\
\hline 7 & - & - & + & Ocasional/Improvisada \\
\hline 8 & - & + & + & Militante \\
\hline
\end{tabular}

Por considerar fundamental a participação integral do pesquisador no compartilhamento das ações em busca de soluções de modo planejado em que a co-gestão é o caminho a ser partilhado, Morin destaca a pesquisa-ação integral como ideal para a aplicação do método de pesquisa-ação porque os pesquisadores privilegiam a pesquisa de campo onde a discussão com todos os atores é enfatizada.

A pesquisa-ação integral é estruturada, de acordo com Morin (2004, p.61), em uma grade de análise do processo com 5 componentes: contrato, participação, mudança, discurso e ação. A aplicação deve ser in loco, dinâmica e sistêmica como é explicada por Morin (2004, p.61): 
A participação é essencial. O contrato é uma das condições que a asseguram. A mudança é a finalidade. Os efeitos dessa participação ou ação negociada estão presentes no discurso ou na transformação dos espíritos e na ação encarada para resolver ou equacionar um problema da melhor maneira possível.

Para melhor entendimento da função de cada um dos 5 componentes da teoria da PAI, utilizamos as explicações de Raphael (2007, p.11-112) no quadro abaixo:

\begin{tabular}{|l|l|}
\hline COMPONENTES & FUNÇÃo \\
\hline Contrato & $\begin{array}{l}\text { O contrato elabora uma linguagem comum, compreensiva a todas as pes- } \\
\text { soas, e apresenta características de negociação e deliberação entre as partes. }\end{array}$ \\
\hline Participação & $\begin{array}{l}\text { A participação deve ser um instrumento que leve à co-gestão e, para tal, } \\
\text { supõe a colaboração de todos os elementos em todas as tomadas de decisão, } \\
\text { em todo o processo. }\end{array}$ \\
\hline Mudança & $\begin{array}{l}\text { A mudança consiste na passagem de um estado a outro, este último o } \\
\text { próprio objetivo do projeto. }\end{array}$ \\
\hline Discurso & $\begin{array}{l}\text { O discurso faz parte da transformação e toma corpo quando inserido na } \\
\text { ação. }\end{array}$ \\
\hline Ação & $\begin{array}{l}\text { A ação atua em espiral com a reflexão, questiona o discurso e deve refletir } \\
\text { o consenso e a capacidade dos participantes. }\end{array}$ \\
\hline
\end{tabular}

Quadro 2: Funções dos componentes da Pesquisa-ação Integrada (PAl)

Existem recomendações que Morin (2004) estabelece para cada um dos componentes cujos âmbitos dimensionam a pesquisa, como por exemplo: que o contrato deve ser aberto, formal e não estruturado porque, se for fechado não faz sentido na pesquisa-ação integral, pois impede questionamentos; que a participação deve visar a co-gestão porque, dentre os três graus de participação - representação, cooperação e co-gestão - visa à participação de todos os membros em todas as tarefas; que a mudança produza transformações na ação e no pensamento porque distingue a pesquisa-ação integral de outros tipos de pesquisa; que o discurso intuitivo ou espontâneo passe cada vez mais ao entendimento esclarecido, consciente e engajado; e que a ação, definidora da pesquisa-ação, seja principalmente coletiva.

A intervenção da pesquisa-ação integral foi realizada, durante reuniões quinzenais, por um grupo denominado "Linguagem UNESP" formado com a pesquisa- 
dora, pesquisadores colaboradores, orientandos de mestrado, doutorado e iniciação científica e catalogadores especializados nas três áreas de conhecimento.

Nas reuniões quinzenais eram desenvolvidos seminários de discussão sobre a atuação profissional na representação e recuperação da informação, compartilhamento de experiências e cursos de capacitação de modo a promover a reflexão crítica com a finalidade de identificar problemas ou avaliar mudanças. Nestes seminários foi apresentada a sistematização teórica e metodológica sobre linguagem documentária e experiências de elaboração de linguagem documentária com uso de softwares a partir das considerações analíticas e comparativas do desenvolvimento da pesquisa.

\section{A linguagem no Manual de política de indexação de bibliotecas universitárias: a "ponta do iceberg" do processo de negociação}

O "Manual da Política de Indexação das bibliotecas universitárias da UNESP” (FUJITA et al., 2014) se compõe de três partes: a definição dos elementos da política de indexação que causam influência na recuperação dos assuntos no catálogo ATHENA, o processo de indexação para a catalogação de assuntos dos documentos das bibliotecas e a linguagem documentária como instrumento de controle de vocabulário para mediar a representação temática na indexação e na estratégia de busca durante a recuperação por assuntos.

A formulação desse manual da política de indexação foi resultado de um processo de negociação que se realizou entre pesquisadores e catalogadores em uma intensa troca de saberes que se iniciaram por ambas as partes, de modo intuitivo e foi, ao longo do tempo, formando um conhecimento que propiciou a descoberta dos problemas, o desenvolvimento de estudos para as ações necessárias às mudanças e à execução do contrato cujo discurso realizou a transformação. $\mathrm{O}$ contrato com o discurso da mudança se concretizou no Manual de política de indexação que é adotado pelas bibliotecas universitárias da UNESP, porém em contínua avaliação pelos grupos mistos de pesquisadores e catalogadores, formados em 2010, de Política de Indexação e Linguagem UNESP.

A proposta de se iniciar a discussão sobre política de indexação partiu do grupo de pesquisa durante curso de capacitação aos catalogadores sobre fundamentos da Indexação para bibliotecas universitárias, realizado em 2009. As discussões iniciadas 
no curso ofereceram aos catalogadores a certeza de que a indexação, com seu corpus teórico e metodológico em representação da informação, instrumentos e avaliação, poderia contribuir para diminuir a obsolescência da catalogação de assuntos e apontar soluções para problemas de recuperação da informação no catálogo online.

O processo de negociação da política realizado com o método de pesquisa-ação integral teve como foco de análise o sistema de recuperação por assuntos do catálogo online ATHENA da rede de bibliotecas universitárias da UNESP. As primeiras observações dos problemas foram obtidas após discussões empíricas baseadas em conhecimento intuitivo e espontâneo dos catalogadores, porém, foram decisivos para o desenvolvimento conjunto de estudos metodologicamente orientados de avaliação.

As primeiras observações levantaram os seguintes problemas:

- a dificuldade na recuperação por assuntos no catálogo online relatada com freqüência por usuários;

- a linguagem utilizada era pouco especializada e por isso era necessário o uso de outras linguagens especializadas e até linguagem natural quando o termo não fosse encontrado.

O levantamento desses problemas motivou a realização de dois estudos de avaliação: de uso da linguagem documentária e de consistência do processo de indexação. Os resultados obtidos foram discutidos pelo grupo e revelaram duas causas importantes: a importação e cópia dos registros bibliográficos impossibilitavam a necessidade de realizarem o processo de catalogação de assuntos e, consequentemente, o uso de linguagem.

Portanto, a falta de uso da linguagem gerava o desconhecimento das potencialidades de seu vocabulário bem como o de obsolescência, tendo em vista que não contribuíam para sua atualização com novos termos. O uso de outras linguagens e até de linguagem natural contribuíam para os problemas de recuperação da informação por assuntos no catálogo online porque não existia um padrão de linguagem para os catalogadores de todas as bibliotecas realizarem a catalogação de assunto e, o mais grave, nem para consulta do usuário durante a estratégia de busca por assuntos.

Com estes aspectos foi possível, na segunda pesquisa de consistência do processo de indexação, demonstrar aos catalogadores que a linguagem documentária, embora desatualizada e pouco especializada, não era a causa principal do proble- 
ma de recuperação por assuntos. A catalogação de assuntos não tinha padrão de procedimentos de análise documental de conteúdos para orientar sua execução sistematizada por todos os catalogadores a exemplo da catalogação descritiva que é regida pelo "Padrão de Qualidade dos Registros Bibliográficos da UNESP” (GATTI, et al., 2002). A falta de procedimentos para a catalogação de assuntos é um grande problema porque impede o uso da linguagem, da avaliação e atualização do vocabulário e, principalmente, da construção de registros de autoridade para a atualização dos campos de assunto no formato MARC21.

As duas explicações são verdadeiras e serviram como as "pontas do iceberg" da proposta da política de indexação para as bibliotecas universitárias da UNESP. Era, portanto, necessária a mudança decidida de comum acordo por todos do grupo e assim foi elaborada a "Proposta de política de indexação para as bibliotecas da Unesp" (FUJITA, et al., 2012). Tal documento contém o contrato que modificou o modo de trabalho e atuação profissional dos catalogadores nas bibliotecas universitárias da UNESP com a introdução no discurso da compreensão de elementos e variáveis de indexação, o processo de indexação e a representação dos termos por meio de uma única linguagem que está em contínua construção e manutenção.

No espírito da participação coletiva para a co-gestão da pesquisa-ação, a "Proposta de política de indexação de bibliotecas universitárias da UNESP” (FUJITA, et al., 2012) foi, antes de ser implantada, apresentada em fevereiro de 2012 para os catalogadores e diretores das bibliotecas e, nessa ocasião aprovada para implantação. A implantação pelo Grupo de Política de Indexação foi realizada com todos os catalogadores por meio de divulgação, capacitação (3 cursos ministrados) e acompanhamento diário orientação e resolução de dúvidas por grupos de áreas de assuntos especializados desde fevereiro de 2010 até o presente.

Após resultados positivos de avaliação de sua aplicabilidade obtidos no período de dois anos, a Proposta tornou-se o "Manual da Política de Indexação das bibliotecas universitárias da UNESP" (FUJITA et al., 2014), um conjunto de condutas, procedimentos e instrumentos imersos em um contexto de gestão e envolvidos pela cultura organizacional da biblioteca.

Pelas características de desenvolvimento da pesquisa e do nível de participação de ambos os grupos, de pesquisadores e de catalogadores, significou que a política de indexação tornou-se uma ação para a transformação. Emanou de uma negociação entre as pessoas que participaram de uma co-gestão, pautados por fundamentação 
teórica e metodológica e respaldados pelo conhecimento mútuo, para promoverem um serviço estratégico com resultados visíveis para a comunidade. Essa co-gestão, assumida por todos, tem consciência dos riscos, problemas e, principalmente, responsabilidade pelas ações de mudanças que dependem inteiramente do trabalho de avaliação, discussão, propostas, implantação e acompanhamento, de modo contínuo.

Neste sentido, é importante observar que a pesquisa-ação integral desenvolveu um produto altamente qualificado de política de indexação cujos resultados são, principalmente, a modificação das condutas de catalogação de assuntos, pois o desenvolvimento do Manual de indexação pautou-se por sua implantação e avaliação, simultaneamente. Isso significa que a política de indexação foi aceita, implantada, avaliada e determinante para a mudança de realidade de atuação profissional dos catalogadores e do catálogo online ATHENA.

Com a decisão de uso de apenas uma linguagem por catalogadores e usuários, o Grupo de Linguagem passou a trabalhar no desenvolvimento da Linguagem UNESP com o objetivo de adequação, manutenção e atualização do vocabulário com a finalidade primordial de disponibilização futura aos usuários para consulta durante busca por assunto no catálogo online.

A adequação, manutenção e atualização do vocabulário são realizadas à medida que avança o processo de indexação. Para isso são consultadas as linguagens documentárias Lista de Cabeçalhos de Assuntos da Rede BIBLIODATA (LCARB), Terminologia de Assuntos da Biblioteca Nacional, Library of Congress Subject Headings (LCSH) e Medical Subject Headings (MeSH) que disponibilizam seus registros de autoridade para exportação.

Esse processo, iniciado em junho de 2012, está em andamento a partir da construção em formato MARC21 de registros de autoridade de assuntos tópicos e geográficos de descritores da linguagem UNESP. Esses procedimentos são etapas da construção da Linguagem UNESP que foram acrescidos com a alteração de termos e inclusão de termos novos conforme procedimentos terminográficos definidos após curso de capacitação "A terminologia na elaboração de linguagem documentária" ministrado ao grupo pelos pesquisadores.

A inclusão de termos novos foi o melhor sinal de que os catalogadores estavam aplicando a política de indexação e, como era previsto, definindo a entrada desses termos em vista da natureza científica e tecnológica e de produção de novos conhecimentos dos documentos que entravam nos acervos das bibliotecas universitárias. 
Ainda não foi possível a disponibilização da linguagem documentária junto à interface de busca, porém, está em desenvolvimento pelo Grupo de Linguagem o estudo da macroestrutura de categorias e subcategorias acompanhadas das relações hierárquicas entre termos com base na LCSH, VocaUSP e Terminologia de Assuntos da Biblioteca Nacional para construção da Linguagem UNESP no modo consulta pelo software do catálogo online. A previsão é que a elaboração dos registros de autoridade obedeça à ordenação e classificação estabelecida por essa macroestrutura disponível na Linguagem UNESP.

\section{Considerações finais}

A indexação na catalogação é a finalidade desta pesquisa para que os catálogos possibilitem a recuperação por assuntos mais precisa e específica, para isso, espera-se que os resultados dessa investigação com o método de pesquisa-ação integrada forneçam às bibliotecas, de um modo geral, uma proposta de um manual de elaboração, implantação e avaliação de linguagem documentária como parte integrante do Manual de política de indexação para assegurar a qualidade da recuperação por assuntos na atual conjuntura de catalogação cooperativa. Por outro lado, que seja também possível o processo de negociação, a co-gestão, o contrato aberto, as mudanças, o discurso engajado e as ações perpassando todo o processo de negociação de forma espiralada e contínua.

A proposta para o futuro é que as bibliotecas se reúnam em sistemas e organizem equipes que possam compartilhar decisões voltadas para a qualidade e consistência da indexação na recuperação de assuntos de seus catálogos baseados em condutas metodológicas que sejam coletivamente desenvolvidas e descritas em manual de indexação a exemplo de serviços de indexação e resumo que funcionam em sistema de cooperação para a produção de bases de dados referenciais.

Com relação às linguagens documentárias, esta investigação demonstra que é possível e necessário os sistemas de bibliotecas universitárias utilizarem linguagens mais estruturadas tais como a LCSH e Terminologia de Assuntos da Biblioteca Nacional e, futuramente, a Linguagem UNESP porque possuem sistemas interoperáveis e disponbilizam seus registros de autoridade para exportação.

Outro aspecto do uso de linguagem documentária é a política de compartilhamento. As bibliotecas universitárias brasileiras poderiam avançar no aprimoramento 
do vocabulário científico e tecnológico brasileiro de modo a permitir estudos bibliométricos e cientométricos mais avançados e, ainda, compartilhar registros de autoridades e inovações tecnológicas na visualização da linguagem para o usuário de modo a possibilitar sua participação na construção e atualização.

Nesse sentido, a experiência de sistemas de bibliotecas universitárias em inserir processos de indexação e adotar uma política de indexação descrita em manual de indexação é importante para estudos de avaliação contínua de recuperação por assuntos. Do mesmo modo que é fundamental a participação dos pesquisadores em Ciência da Informação no desenvolvimento de pesquisas dedicadas ao aprimoramento de catálogos on-line no que tange ao processo de análise e representação de assuntos para a recuperação e também para estudos métricos ligados à visibilidade científica brasileira e internacional.

Com a sistematização teórica e metodológica sobre indexação e linguagem documentária em uma política de indexação, entendemos ser imprescindível, do ponto de vista educacional, que o catalogador em sua formação inicial e continuada possa ter conhecimento do contexto de catalogação em bibliotecas, examinando os procedimentos, instrumentos, regras e condutas utilizadas na análise e representação de assuntos de livros e demais recursos informacionais pela indexação com auxílio de uma linguagem documentária representativa de domínios de assuntos especializados e atualizada, conforme observação contínua do funcionamento do catálogo pela recuperação por assunto e comportamento informacional do usuário. Nesse sentido, consideramos que a pesquisa fornecerá subsídios para que a indexação como processo de análise e representação de assuntos não seja dissociada do ambiente de bibliotecas nos conteúdos curriculares.

Acreditamos que a pesquisa de observação participante e a pesquisa-ação, metodologias desenvolvidas nesta pesquisa, propiciaram as diferentes perspectivas dos participantes do contexto de indexação, usuários, catalogadores, dirigentes de bibliotecas e bibliotecários, assim como revelaram claramente o que pensam sobre os problemas e restrições desse contexto, como seria possível resolvê-los e quais os instrumentos e métodos necessários, o que ajudou no aprimoramento da política de indexação. Mais do que isso, deu aos catalogadores a possibilidade de compreensão e, conseqüente domínio, de como desenvolver a organização e representação do conhecimento no contexto de bibliotecas universitárias. 


\section{Referências}

DESCOMBE, M. The good research guide. 2. ed. Buckingham: Open University Press, 2005.310p.

DESROCHE, H. Entrependre d'apprendre: d'une autobiographie raisonnée aux projets d'une recherche-action. Paris: Ouvrières, 1990.

FUIITA, M. S. L. et al. Proposta de política de indexação para as bibliotecas universitárias da UNESP. Marília: Faculdade de Filosofia e Ciências, UNESP; São Paulo: Coordenadoria Geral de Bibliotecas, Reitoria da UNESP, 2012. FUJITA, M. S. L. et al. Manual da política de indexação para as bibliotecas universitárias da UNESP. Marília: Faculdade de Filosofia e Ciências, UNESP; São Paulo: Coordenadoria Geral de Bibliotecas, Reitoria da UNESP, 2014. Disponível em: <http://www.biblioteca.unesp.br/portal/arquivos/manual-politica-indexacao/> FUJITA, M. S. L., RUBI, M. P., BOCCATO, V. R. C. O contexto sociocognitivo do catalogador em bibliotecas universitárias: perspectivas para uma política de tratamento da informação documentária. Datagramazero - Revista de Ciência da Informação, v.10, 2009. Recuperado de http://www.dgz.org.br/.

FUJITA, M. S. L., BOCCATO, V. R. C., RUBI, M. P. A política de indexação elaborada por catalogadores: um estudo de observação participante em cursos de capacitação. In: A. Pulgarín Guerrero, Antonio; A. Vivas Moreno (Org.). Limites, fronteras y espacios comunes: encuentros y desencuentros en las ciencias de la información. Badajoz: Universidad de Extremadura, 2012. pp. 90-101

GATTI, C. A. de S. et al. Padrão de qualidade de registros bibliográficos da Unesp. Marilia: Universidade Estadual Paulista; Coordenadoria Geral de Bibliotecas, 2002. 2 v.

LANCASTER, F. W. Indexação e resumos: teoria e prática. Tradução de Antonio Agenor Briquet de Lemos. 2. ed. Brasília: Briquet de Lemos, 2004.

LIMA, J. A. de O. Pesquisa-ação em ciência da informação. In: MUELLER, S. P. M. (Org.) Métodos para a pesquisa em ciência da informação. Brasília:Thesaurus, 2007. p. 63-82.

LIMA, V. M. A.; BOCCATO, V. R. C. O desempenho terminológico dos descritores em Ciência da Informação do Vocabulário Controlado do SIBi/USP nos processos de indexação manual, automática e semi-automática. Perspect. ciênc. inf., Belo Horizonte, v. 14, n. 1, Apr. 2009. Available from <http://www.scielo.br/scielo.php?script=sci_arttext\&pid=S1413-99362009000100010\&lng=en\&nrm=iso $>$. access on 14 June 2014. http://dx.doi. org/10.1590/S1413-99362009000100010.

MORIN, A. Pesquisa-ação integral e sistêmica: uma antropedagogia renovada. Trad. de Michel Thiollent. Rio de Janeiro: DP\&A, 2004. 232p.

RAPHAEL, H. S. Pesquisa ação. In: MACHADO, L. M. M., MAIA, G. Z. A., LABEGALINI, A. C. F. B. Pesquisa em educação: passo a passo. Marília: Edições M3T, 2007. p.105-116.

THIOLLENT, M. Metodologia pesquisa-ação. 8. ed. São Paulo: Cortez, 1998. 108 p.

17 | Revista Conhecimento em Ação, Rio de Janeiro, v. 1, n. 1, jan/jun. 2016 